\title{
Cluster physics from joint weak gravitational lensing and Sunyaev-Zel'dovich data
}

\author{
O. Doré ${ }^{1}$, F. R. Bouchet ${ }^{1}$, Y. Mellier ${ }^{1,2}$, and R. Teyssier ${ }^{3,1,4}$ \\ 1 Institut d'Astrophysique de Paris, 98bis boulevard Arago, 75014 Paris, France \\ 2 Observatoire de Paris, DEMIRM, 61 avenue de l'Observatoire, 75014 Paris, France \\ 3 Service d'Astrophysique, DAPNIA, Centre d'Études de Saclay, 91191 Gif-sur-Yvette, France \\ 4 Numerical Investigations in Cosmology (NIC) group, CEA Saclay, France
}

Received 9 February 2001 / Accepted 16 May 2001

\begin{abstract}
We present a self consistent method to perform a joint analysis of Sunyaev-Zel'dovich and weak gravitational lensing observation of galaxy clusters. The spatial distribution of the cluster main components is described by a perturbative approach. Assuming hydrostatic equilibrium and the equation of state, we are able to deduce, from observations, maps of projected gas density and gas temperature. The method then naturally entails a X-ray emissivity prediction which can be compared to observed X-ray emissivity maps. By comparing with 2 simulated clusters we find that this prediction turns out to be in very good agreement with the simulations. The simulated and predicted surface brightness images have a correlation coefficient higher than 0.9 and their total flux differs by $0.9 \%$ or $9 \%$. The method should be able to deal with real data in order to provide a physical description of the state of the cluster and of its constituents. The tests performed show that we can recover the amount and the spatial distributions of both the baryonic and non-baryonic material with an accuracy better than $10 \%$. So, in principle, it might indeed help to alleviate some well known biases affecting, e.g. baryon fraction measurements.
\end{abstract}

Key words. gravitational lensing - galaxies: clusters: general - cosmology: observations - X-rays: galaxies

\section{Introduction}

Whereas clusters of galaxies, as the largest gravitationaly bound structures in the universe, are a natural probe of cosmology, observations, numerical simulations as well as timing arguments provide compelling evidence that most clusters are young and complex systems. Interaction with large-scale structures, merging processes and coupling of dark matter with the intra-cluster medium complicate the interpretation of observations and the modeling of each components. Since clusters are composed of dark matter (DM), galaxies and a hot dilute X-ray emitting gas (Intra cluster medium, ICM) accounting respectively for $\sim 85 \%$, $\sim 10 \%$ and $\sim 5 \%$ of their mass, the physics of the ICM bounded in a dark matter gravitational potential plays a major role in cluster formation and evolution.

Each component can be observed in many different ways. In particular, we refer to gravitational lensing effects (the weak-lensing regime here, WL) (Mellier 2000; Bartelmann \& Schneider 2001), the Sunyaev-Zel'dovich (SZ) effect (Sunyaev \& Zel'dovich 1972; Birkinshaw 1999) and X-ray emission (X) (Sarrazin 1988). Whereas the former probes mostly the dark matter component, both the latter probe the baryons of the gravitationally bound ICM.

Send offprint requests to: O. Doré, e-mail: dore@iap.fr
Due to observational progress, increasingly high quality data are available that enable multi-wavelength investigation of clusters down to an arc-minute scale (the most recent is the spectacular progress in SZ measurements, e.g. Reese et al. (2000) and Désert et al. (1998). In contrast with past decades, it is now possible to analyse clusters of galaxies using X-ray, WL and SZ data at almost the same resolution, and to produce from them self consistent models. The exploration of reconstruction techniques to analyse together these high quality data sets and exploit their complementarity is a recent challenge. It has been tackled by several groups (Zaroubi et al. 1998; Grego et al. 1999; Reblinsky \& Bartelmann 1999; Zaroubi et al. 2000; Castander et al. 2000; Holder et al. 2000). Zaroubi et al. and Reblinsky et al. attempted a full deprojection by assuming isothermality and axial symmetry, using respectively a least square minimization and a Lucy-Richardson algorithm. Grego et al. compare SZ-derived gas mass to WL-derived total mass by fitting a spheroidal $\beta$ model. Whereas these methods give reasonable results it has been pointed out, e.g. by Inagaki et al. (1995) in the context of $H_{0}$ measurement from SZ and X-ray observations, that both non-isothermality and asphericity analysis can trigger systematic errors as high as $20 \%$.

In this paper, we propose an alternative direction in order to to get rid of both isothermal and spherical hypotheses. Our method is based on a self-consistent use of 
WL and SZ observables, and on a perturbative development of general physical hypotheses. This approach allows us to test some very general physical hypotheses of the gas (hydrostatic equilibrium, global thermodynamic equilibrium) and also naturally provides some $\mathrm{X}$ observation predictions.

Observations only provide $2-D$ projected quantities (e.g. mass, gas pressure, ...). These quantities are related to each other by physical hypotheses which express the $3-D$ geometrical properties and the dynamical and thermodynamical stage of matter (e.g. sphericity, hydrostatic equilibrium, equation of state). In most cases, their 3-D mathematical formulations do not have any tractable equivalent relating $2-D$ projected quantities: in particular, projection along the line of sight does not provide an equation of state or a projected hydrostatic equilibrium equation. Therefore, as soon as we want to compare these data (WL, SZ, X) we have to deproject the relevant physical quantities $\left(P_{\mathrm{g}}, T_{\mathrm{g}}, \rho_{\mathrm{g}} \ldots\right)$. This can be done only using strong assumptions, either by using parametric models (e.g. a $\beta$ model, Cavaliere \& Fusco-Femaino 1976) or by assuming mere geometrical hypotheses (the former necessarily encompassing the latter) (Fabian et al. 1981; Yoshikawa \& Suto 1999).

Because the latter keeps the physical grounds as general as possible and also avoids as many theoretical biases as possible, we preferred to focus on the geometric approach. Furthermore, this simplest choice is also motivated by looking at some images of observed clusters (Désert et al. 1998; Grego et al. 1999). Some do have striking regularity with almost circular or ellipsoidal appearance, as we expect for fully relaxed systems. Then, since relaxed clusters are expected to be spheroidal in favored hierarchical structure formation scenario, it is natural to try to relate the observed quasi-circularity (quasi-sphericity) to the $3-D$ quasi-sphericity (quasi-spheroidality). We perform this by using some linearly perturbed spherical (spheroidal) symmetries in a self-consistent approach.

The paper is organised as follows: in Sect. 2 we define our physical hypothesis and notations. The method is precisely described in Sect. 3. We consider both the spherical as well as spheroidal cases and obtain a predicted $\mathrm{X}$ surface brightness map from a SZ decrement map and a WL gravitational distortion map. In Sect. 4 a demonstration with simulated clusters is presented before discussing its application to genuine data as well as perspectives for further developments in Sect. 5 .

\section{Hypotheses, Sunyaev-Zel'dovich effect and the weak lensing}

We now briefly describe our notations as well as our physical hypothesis.

\subsection{General hypotheses}

Following considerations fully detailed in Sarrazin (1988) the ICM can be regarded as a hot and dilute plasma constituted by ions and electrons, whose respective kinetic temperatures $T_{\mathrm{p}}$ and $T_{\mathrm{e}}$ will be considered as equal $T_{\mathrm{p}}=T_{\mathrm{e}} \equiv T_{\mathrm{g}}$. This is the global thermodynamic equilibrium hypothesis which is expected to hold up to $r_{\text {virial }}$ (see Teyssier et al. 1997 and Chièze et al. 1998 for a precise discussion). Given the low density (from $n_{\mathrm{e}} \sim 10^{-1} \mathrm{~cm}^{-3}$ in the core to $\sim 10^{-5} \mathrm{~cm}^{-3}$ in the outer part) and high temperature of this plasma $(\sim 10 \mathrm{keV})$, it can be treated as a perfect gas satisfying the equation of state:

$P_{\mathrm{g}}=\frac{\rho_{\mathrm{g}} k_{\mathrm{B}} T_{\mathrm{g}}}{\mu_{\mathrm{e}} m_{\mathrm{p}}}=\beta \rho_{\mathrm{g}} T_{\mathrm{g}}$

with $\beta \equiv \frac{k_{\mathrm{B}}}{\mu_{\mathrm{e}} m_{\mathrm{p}}}$. Let us consider in accord with the general paradigm that most of the mass is constitued by a dark matter component and thus neglect the gas mass with regards to it, and let us assume stationarity (no gravitational potential variation on a time scale smaller than the hydrodynamic time scale, e.g. no recent mergers). Then the gas assumed to be in hydrostatic equilibrium in the dark matter gravitational potential satisfies:

$\nabla\left(\rho_{\mathrm{g}} \boldsymbol{v}_{\mathrm{g}}\right)=0$

$\nabla P_{\mathrm{g}}=-\rho_{\mathrm{g}} \nabla \Phi_{\mathrm{DM}}$.

At this point there is no need to assume isothermality.

\subsection{Sunyaev-Zel'dovich effect and weak lensing}

Inverse Compton scattering of cosmic background (CMB) photons by the electrons in the ICM modifies the CMB spectrum (Zel'dovich \& Sunyaev 1969; Sunyaev \& Zel'dovich 1972; Sunyaev \& Zel'dovich 1980). The amplitude of the SZ temperature decrement $\frac{\Delta T_{\mathrm{SZ}}}{T_{\mathrm{CMB}}}$ is directly proportional to the Comptonisation parameter $y$ which is given by:

$$
\begin{aligned}
y & =\frac{\sigma_{\mathrm{T}}}{m_{\mathrm{e}} c^{2}} \int \mathrm{d} l n_{\mathrm{e}} k_{\mathrm{B}} T_{\mathrm{e}}=\frac{\sigma_{\mathrm{T}}}{m_{\mathrm{e}} c^{2}} \int \mathrm{d} l p_{\mathrm{e}} \\
& =\frac{\sigma_{\mathrm{T}}}{m_{\mathrm{e}} c^{2}} \int \mathrm{d} l \frac{\rho_{\mathrm{g}} k_{\mathrm{B}} T_{\mathrm{g}}}{\mu_{\mathrm{e}} m_{\mathrm{p}}}=\alpha \int \mathrm{d} l P_{\mathrm{g}},
\end{aligned}
$$

where $\alpha \equiv \frac{\sigma_{\mathrm{T}}}{m_{\mathrm{e}} c^{2}}, k_{\mathrm{B}}$ is the Boltzmann's constant, $\sigma_{\mathrm{T}}$ is the Thomson scattering cross-section and $\mathrm{d} l$ is the physical line-of-sight distance. $m_{\mathrm{e}}, n_{\mathrm{e}}, T_{\mathrm{e}}$ and $p_{\mathrm{e}}$ are the mass, the number density, the temperature and the thermal pressure of electrons. $\rho_{\mathrm{g}}$ and $T_{\mathrm{g}}$ respectively denote the gas density and temperature, and $\mu_{\mathrm{e}}$ is the number of electrons per proton mass. Some further corrections to this expression can be found in Rephaeli (1995) and Birkinshaw (1999).

In parallel to this spectral distortion, the statistical determination of the shear field $\gamma$ affecting the images of background galaxies enables us to derive the dominant projected gravitational potential of the lens produced by dark matter $(\mathrm{DM}): \phi_{\mathrm{DM}}$ in our general hypothesis (see Mellier 2000 for details). 


\section{Method}

\subsection{Principle}

Let us suppose we have for a given cluster a set of SZ and WL data which enables us to construct a $2-D$ map of projected gas pressure as well as a $2-D$ projected gravitational potential map. We now propose an answer to the following question: how should we co-analyze these various data sets? Our first aim is to develop a method which allows us to get maps of projected thermodynamical quantities with as few physical hypotheses as possible.

Our method is the following. Let us suppose then that these maps have almost a rotationnal symmetry, as is the case for a vast class of experimental observations as e.g. in Fig. 1. More precisely, let us suppose that the projected gas pressure $y$ as well as the observed projected gravitational potential $\phi_{\mathrm{DM}}$ can be well fitted by the following type of functions:

$y(R, \varphi)=y_{0}(R)+\varepsilon y_{1}(R) m(\varphi)$

$\phi_{\mathrm{DM}}(R, \varphi)=\phi_{\mathrm{DM}, 0}(R)+\varepsilon \phi_{\mathrm{DM}, 1}(R) n(\varphi)$

where $\varepsilon \ll 1,(R, \varphi)$ denotes polar coordinates in the image plane and $m$ and $n$ are some particular functions. This description means first of all that the images we see are linear perturbations from some perfect circularly symmetric images, and second that the perturbation might be described conveniently by the product of a radial function and an angular function. Equivalently, we can assert that to zeroth order in $\varepsilon$ our images are circularly symmetric but they admit some corrections to a first order in $\varepsilon$.

We then assume that these observed perturbed symmetries are a consequence of an intrinsic 3-D spherical symmetry linearly perturbed as well. This point constitutes our key hypothesis. It means that to the zeroth order in a certain parameter (e.g. $\varepsilon$ ) our clusters are regular objects with a strong circular symmetry but they admit some first order (linear) perturbations away from this symmetry, whose angular part is separable. As a consequence of these assumptions we will make use of this linearly perturbed symmetry to get a map of some complementary projected thermodynamical quantities, the gas density $D_{\mathrm{g}}$ and the gas temperature $\zeta_{\mathrm{g}}$, successively to zeroth and first order in $\varepsilon$.

Formulated this way, the problem yields a natural protocol:

- Looking at some maps with this kind of symmetry, we compute a zeroth order map $\left(y_{0}(R), \phi_{0}(R)\right)$ with a perfect circular symmetry by averaging over some concentric annulus. A correction for the bias introduced by perturbations is included. These zeroth order quantities allow us to derive some zeroth order maps of $D_{\mathrm{g}, 0}(R)$ and $\zeta_{\mathrm{g}, 0}(R)$ with a perfect circular symmetry;

- We then take into account the first order corrections to this perfect symmetry $\left(y_{1}(R) m(\varphi), \phi_{1}(R) m(\varphi)\right)$ and infer from them first order correction terms to the zeroth order maps: $D_{\mathrm{g}, 1}(R, \varphi)$ and $\zeta_{\mathrm{g}, 1}(R, \varphi)$.
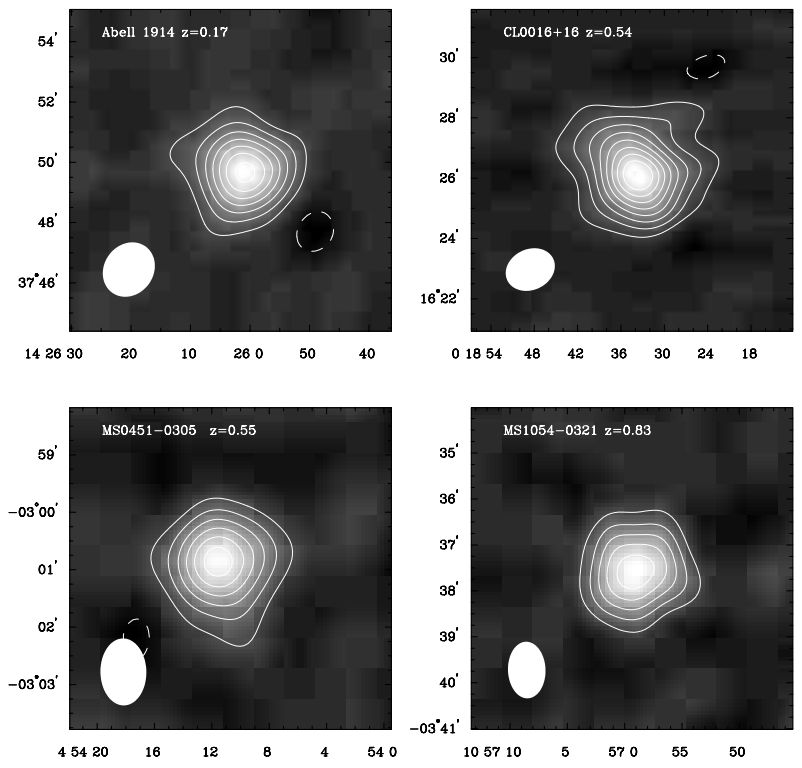

Fig. 1. Images of the SZ effect observed towards four galaxy clusters with various redshifts. The contours correspond to 1.5 to 5 times the noise level. Data were obtained with the lownoise $\mathrm{cm}$-wave receiver installed on the OVRO and BIMA mmwave interferometric arrays (Holder \& Carlstrom 1999). The bottom-left white ellipse denotes the synthetised beam for each observation.

Even if, for the sake of clarity, we formulate our method assuming a perturbed circular symmetry, it applies equivalently to a perturbed elliptical symmetry, as will be shown below. In this more general case, we assume that the cluster exhibits a linearly perturbed spheroidal symmetry.

\subsection{The spherically symmetric case: From observations to predictions}

Let us now apply the method to the case where the projected gas density (SZ data) and the projected gravitational potential (WL data) look almost circular. These observations lead us to suppose that the $3-D$ gas pressure, the gravitational potential, the gas density and the gas temperature can be well described by the following equations:

$$
\left\{\begin{array}{cccc}
P_{\mathrm{g}}(r, \theta, \varphi) & =P_{\mathrm{g}, 0}(r)+\varepsilon & P_{\mathrm{g}, 1}(r) f(\theta, \varphi) \\
\Phi_{\mathrm{DM}}(r, \theta, \varphi) & =\Phi_{\mathrm{DM}, 0}(r)+\varepsilon & \Phi_{\mathrm{DM}, 1}(r) g(\theta, \varphi) \\
\rho_{\mathrm{g}}(r, \theta, \varphi) & =\rho_{\mathrm{g}, 0}(r)+\varepsilon & \rho_{\mathrm{g}, 1}(r) h(\theta, \varphi) \\
T_{\mathrm{g}}(r, \theta, \varphi) & =T_{\mathrm{g}, 0}(r)+\varepsilon & T_{\mathrm{g}, 1}(r) k(\theta, \varphi)
\end{array}\right.
$$

where $(r, \theta, \varphi)$ are spherical coordinates centered on the cluster.

\subsubsection{The hydrostatic equilibrium}

If we first apply the hydrostatic equilibrium equation $\nabla P_{\mathrm{g}}=-\rho_{\mathrm{g}} \nabla \Phi_{\mathrm{DM}}$ we get the following equations. To zeroth order in $\varepsilon$ we have

$P_{\mathrm{g}, 0}^{\prime}(r)=-\rho_{\mathrm{g}, 0}(r) \Phi_{\mathrm{DM}, 0}^{\prime}(r)$, 
and to first order in $\varepsilon$ :

$$
\begin{aligned}
P_{\mathrm{g}, 1}^{\prime}(r) f(\theta, \varphi)= & -\rho_{\mathrm{g}, 0}(r) \Phi_{\mathrm{DM}, 1}^{\prime}(r) g(\theta, \varphi) \\
& -\rho_{\mathrm{g}, 1}(r) \Phi_{\mathrm{DM}, 0}^{\prime}(r) h(\theta, \varphi)
\end{aligned}
$$

$P_{\mathrm{g}, 1}(r) \partial_{\theta} f(\theta, \varphi)=-\rho_{\mathrm{g}, 0}(r) \Phi_{\mathrm{DM}, 1}(r) \partial_{\theta} g(\theta, \varphi)$

$P_{\mathrm{g}, 1}(r) \partial_{\varphi} f(\theta, \varphi)=-\rho_{\mathrm{g}, 0}(r) \Phi_{\mathrm{DM}, 1}(r) \partial_{\varphi} g(\theta, \varphi)$

where " I" denotes the derivative with regards to $r$. Combining Eqs. (11) and (12) we get

$f(\theta, \varphi)=\lambda_{1} g(\theta, \varphi)+\lambda_{2}$

where $\lambda_{1,2}$ are constants. Then, by using Eq. (10) we can write

$f(\theta, \varphi)=\gamma_{1} h(\theta, \varphi)+\gamma_{2}$

where $\gamma_{1,2}$ are constants as well. At this point, we can get rid of $\lambda_{2}$ and $\gamma_{2}$ by absorbing them in the zeroth order mere radial term (i.e. $\rho_{\mathrm{g}, 0}(r)$ and $\left.\Phi_{\mathrm{DM}, 0}(r)\right)$. This means we can consider $\lambda_{2}=0$ and $\gamma_{2}=0$. Similarly, we choose to rescale $\rho_{\mathrm{g}, 1}(r)$ and $\Phi_{\mathrm{DM}, 1}(r)$ so that we can take $\gamma_{1}=$ $\lambda_{1}=1$. These simple equalities lead us to assume from now on:

$f(\theta, \varphi)=h(\theta, \varphi)=g(\theta, \varphi)$.

This is in no way a restriction since it simply means that we absorb integration constants by redefining some terms. This is possible since the relevant part of $f$ (and thus $h$ ) will be fitted on observations, as will be shown below. Taking Eq. (15) into account, Eqs. (10)-(12) simplify to:

$P_{\mathrm{g}, 0}^{\prime}(r)=-\rho_{\mathrm{g}, 0}(r) \Phi_{\mathrm{DM}, 0}^{\prime}(r)$

$P_{\mathrm{g}, 1}^{\prime}(r)=-\rho_{\mathrm{g}, 0}(r) \Phi_{\mathrm{DM}, 1}^{\prime}(r)-\rho_{\mathrm{g}, 1}(r) \Phi_{\mathrm{DM}, 0}^{\prime}(r)$

$P_{\mathrm{g}, 1}(r)=-\rho_{\mathrm{g}, 0}(r) \Phi_{\mathrm{DM}, 1}(r)$.

\subsubsection{The equation of state}

We have now identified the angular part to the first order correction of $P_{\mathrm{g}}, \Phi_{\mathrm{DM}}$ and $\rho_{\mathrm{g}}$. We still have to link those quantities to the angular dependent part of the temperature $T_{\mathrm{g}}$, namely $k(\theta, \varphi)$. This is done naturally by using the equation of state (1), which directly provides, to zeroth and first order in $\varepsilon$ :

$P_{\mathrm{g}, 0}(r)=\beta \rho_{\mathrm{g}, 0}(r) T_{\mathrm{g}, 0}(r)$

$$
\begin{aligned}
P_{\mathrm{g}, 1}(r) f(\theta, \varphi)= & \beta \rho_{\mathrm{g}, 1}(r) T_{\mathrm{g}, 0}(r) f(\theta, \varphi) \\
& +\beta \rho_{\mathrm{g}, 0}(r) T_{\mathrm{g}, 1}(r) k(\theta, \varphi) .
\end{aligned}
$$

This last equation leads naturally to $f(\theta, \varphi)=k(\theta, \varphi)$ if we decide once again to absorb any multiplicative factor in the radial part. This way we see that our choice of separating the radial and angular part is in no way a restriction. We eventually get

$P_{\mathrm{g}, 0}(r)=\beta \rho_{\mathrm{g}, 0}(r) T_{\mathrm{g}, 0}(r)$

$P_{\mathrm{g}, 1}(r)=\beta \rho_{\mathrm{g}, 1}(r) T_{\mathrm{g}, 0}(r)+\beta \rho_{\mathrm{g}, 0}(r) T_{\mathrm{g}, 1}(r)$.

\subsubsection{The observations}

Given this description of the cluster physical state, the experimental SZ and WL data provide the projected quantities $y(R, \varphi)$ and $\phi_{\mathrm{DM}}(R, \varphi)$ :

$$
\begin{aligned}
y(R, \varphi) & =\alpha \int P_{\mathrm{g}, 0}(r) \mathrm{d} l+\varepsilon \alpha \int P_{\mathrm{g}, 1}(r) f(\theta, \varphi) \mathrm{d} l \\
& \equiv y_{0}(R)+\varepsilon y_{1}(R) m(\varphi) \\
\phi_{\mathrm{DM}}(R, \varphi) & =\int \Phi_{\mathrm{DM}, 0}(r) \mathrm{d} l+\varepsilon \int \Phi_{\mathrm{DM}, 1}(r) f(\theta, \varphi) \mathrm{d} l \\
& \equiv \phi_{\mathrm{DM}, 0}(R)+\varepsilon \phi_{\mathrm{DM}, 1}(R) m(\varphi) .
\end{aligned}
$$

Note that in order to get this set of definitions we choose the polar axis of the cluster along the line of sight so that the same azimuthal angle $\varphi$ is used for $2-D$ and $3-D$ quantities.

Our aim is now to derive both a projected gas density map and projected temperature map as defined by:

$$
\begin{aligned}
D_{\mathrm{g}}(R, \varphi) & =\int \rho_{\mathrm{g}}(r, \varphi) \mathrm{d} l \\
& =\int \rho_{\mathrm{g}, 0}(r) \mathrm{d} l+\varepsilon \int \rho_{\mathrm{g}, 1}(r) f(\theta, \varphi) \mathrm{d} l \\
& \equiv D_{\mathrm{g}, 0}(R)+D_{\mathrm{g}, 1}(R, \varphi) \\
\zeta_{\mathrm{g}}(R, \varphi) & =\int T_{\mathrm{g}}(r, \varphi) \mathrm{d} l \\
& =\int T_{g, 0}(r) \mathrm{d} l+\varepsilon \int T_{\mathrm{g}, 1}(r) f(\theta, \varphi) \mathrm{d} l \\
& \equiv \zeta_{\mathrm{g}, 0}(R)+\zeta_{\mathrm{g}, 1}(R, \varphi)
\end{aligned}
$$

\subsubsection{A projected gas density map to zeroth order...}

Now that we have expressed our observables in terms of $3-D$ physical quantities, it is easy to infer a gas density map successively to zeroth and first order in $\varepsilon$. To zeroth order the hydrostatic equilibrium condition (9) states that

$$
P_{\mathrm{g}, 0}^{\prime}(r)=-\rho_{\mathrm{g}, 0}(r) \Phi_{\mathrm{DM}, 0}^{\prime}(r) .
$$

In order to use it we need to deproject the relevant quantities. From the well-known spherical deprojection formula (Binney \& Tremaine 1987) based on Abel's transform we have:

$$
\begin{aligned}
\alpha P_{\mathrm{g}, 0}(r) & =-\frac{1}{\pi} \int_{r}^{\infty} y_{0}^{\prime}(R) \frac{\mathrm{d} R}{\left(R^{2}-r^{2}\right)^{\frac{1}{2}}} \\
& =-\frac{1}{\pi} \int_{0}^{\infty} y_{0}^{\prime}(r \cosh u) \mathrm{d} u
\end{aligned}
$$

where $R=r \cosh u$. Thus, we can write

$$
\begin{aligned}
\alpha P_{\mathrm{g}, 0}^{\prime}(r) & =-\frac{1}{\pi} \int_{0}^{\infty} \cosh u y^{\prime \prime}(r \cosh u) \mathrm{d} u \\
& =-\frac{1}{\pi} \int_{r}^{\infty} \frac{1}{r} \frac{R}{\left(R^{2}-r^{2}\right)^{\frac{1}{2}}} y_{0}^{\prime \prime}(R) \mathrm{d} R .
\end{aligned}
$$

Similarly,

$\Phi_{\mathrm{DM}, 0}^{\prime}(r)=-\frac{1}{\pi} \int_{r}^{\infty} \frac{1}{r} \frac{R}{\left(R^{2}-r^{2}\right)^{\frac{1}{2}}} \phi_{0}^{\prime \prime}(R) \mathrm{d} R$. 
We then get for the projected gas density

$$
\begin{aligned}
& D_{\mathrm{g}, 0}(R)=-2 \int_{R}^{\infty} \frac{r \mathrm{~d} r}{\left(r^{2}-R^{2}\right)^{\frac{1}{2}}} \frac{P_{\mathrm{g}, 0}^{\prime}(r)}{\Phi_{\mathrm{DM}, 0}^{\prime}(r)} \\
& \quad=-\frac{2}{\alpha} \int_{R}^{\infty} \frac{r \mathrm{~d} r}{\left(r^{2}-R^{2}\right)^{\frac{1}{2}}}\left(\frac{\int_{r}^{\infty} \frac{s \mathrm{~d} s}{r\left(s^{2}-r^{2}\right)^{\frac{1}{2}}} y_{0}^{\prime \prime}(s)}{\int_{r}^{\infty} \frac{s \mathrm{~d} s}{r\left(s^{2}-r^{2}\right)^{\frac{1}{2}}} \phi_{0}^{\prime \prime}(s)}\right) .
\end{aligned}
$$

\subsection{5. ... and a projected gas temperature map to zeroth order}

Once we build this projected gas density map, we can recover the projected gas temperature map. If we apply the equation of state (19) we get:

$$
\begin{aligned}
\zeta_{\mathrm{g}, 0}(R) & =\frac{1}{\beta} \int \frac{P_{\mathrm{g}, 0}(r)}{\rho_{\mathrm{g}, 0}(r)} \mathrm{d} l \\
& =-\frac{1}{\beta} \int \frac{P_{\mathrm{g}, 0}(r)}{P_{\mathrm{g}, 0}^{\prime}(r)} \Phi_{\mathrm{DM}, 0}^{\prime}(r) \mathrm{d} l \\
& =-\frac{1}{\pi \beta} \int_{R}^{\infty} \frac{P_{\mathrm{g}, 0}(r)}{P_{\mathrm{g}, 0}^{\prime}(r)} \Phi_{\mathrm{DM}, 0}^{\prime}(r) \frac{r \mathrm{~d} r}{\left(r^{2}-R^{2}\right)^{\frac{1}{2}}} .
\end{aligned}
$$

Since all the required functions $\left(P_{\mathrm{g}, 0}, P_{\mathrm{g}, 0}^{\prime}, \Phi_{\mathrm{DM}, 0}^{\prime}\right)$ have been derived in the previous section (Eqs. (33) and (35)) we can get in this way a projected gas temperature map.

\subsubsection{Corrections from departure to spherical symmetry: a projected gas density map to first order...}

We now reach the core of our method, namely we aim at deriving the quantity $D_{\mathrm{g}, 1}$ defined by (27), i.e. the first order correction to the perfectly circular term :

$$
\begin{aligned}
D_{\mathrm{g}}(R, \varphi) & =D_{\mathrm{g}, 0}(R)+\varepsilon D_{\mathrm{g}, 1}(R, \varphi) \\
& =\int \rho_{\mathrm{g}, 0}(r) \mathrm{d} l+\varepsilon \int \rho_{\mathrm{g}, 1}(r) f(\theta, \varphi) \mathrm{d} l .
\end{aligned}
$$

If we derive Eq. (18) and combine it with Eq. (17) we note that

$\rho_{\mathrm{g}, 0}^{\prime}(r) \Phi_{\mathrm{DM}, 1}(r)=\rho_{\mathrm{g}, 1}(r) \Phi_{\mathrm{DM}, 0}^{\prime}(r)$.

Therefore we can write

$\int \rho_{\mathrm{g}, 1}(r) f(\theta, \varphi) \mathrm{d} l=\int \frac{\rho_{\mathrm{g}, 0}^{\prime}(r)}{\Phi_{\mathrm{DM}, 0}^{\prime}(r)} \Phi_{\mathrm{DM}, 1}(r) f(\theta, \varphi) \mathrm{d} l$.

At this point we want to express this quantity either in terms of WL data or in terms of SZ data depending on their quality, or even better, in terms of an optimal combination of them.

WL data provide straightforward access to the function $\phi_{1}(R) m(\varphi)=\int \Phi_{\mathrm{DM}, 1}(r) f(\theta, \varphi) \mathrm{d} l$ thus we choose to approximate (45) by

$$
\begin{aligned}
& \int \rho_{\mathrm{g}, 1}(r) f(\theta, \varphi) \mathrm{d} l \simeq \frac{\rho_{\mathrm{g}, 0}^{\prime}(R)}{\Phi_{\mathrm{DM}, 0}^{\prime}(R)} \int \Phi_{\mathrm{DM}, 1}(r) f(\theta, \varphi) \mathrm{d} l \\
& \simeq \simeq \frac{\rho_{\mathrm{g}, 0}^{\prime}(R)}{\Phi_{\mathrm{DM}, 0}^{\prime}(R)} \phi_{1}(R) m(\varphi) \\
& \simeq \frac{\rho_{\mathrm{g}, 0}^{\prime}(R)}{\Phi_{\mathrm{DM}, 0}^{\prime}(R)}\left(\phi_{\mathrm{DM}}(R, \varphi)-\phi_{0}(R)\right)
\end{aligned}
$$

where we used the definitions of Sect. 3.2.3 and where $R$ corresponds to the radius observed in the image plane, i.e. the radius $r$ equal to the distance between the line of sight and the center of the cluster. We will discuss this approximation in more detail in Sect. 3.2.8 and validate it through practical implementation on simulations in Sect. 4 . We already can make the following statements: if the line of sight follows a line of constant $r$ throughout the domain of the perturbation, this expression would be rigorously exact. Moreover, it turns out to be a good approximation because of the finite extent of the perturbation.

On the other hand, SZ data provide a measurement of the function $y_{1}(R) m(\varphi)=\int P_{\mathrm{g}, 1}(r) f(\theta, \varphi) \mathrm{d} l$ therefore, we can use Eqs. (18) and (16) to write

$$
\begin{aligned}
& \int \rho_{\mathrm{g}, 1}(r) f(\theta, \varphi) \mathrm{d} l=\int \frac{\rho_{\mathrm{g}, 0}^{\prime}(r)}{P_{\mathrm{g}, 0}^{\prime}(r)} P_{\mathrm{g}, 1}(r) f(\theta, \varphi) \mathrm{d} l \\
& \simeq \frac{\rho_{\mathrm{g}, 0}^{\prime}(R)}{P_{\mathrm{g}, 0}^{\prime}(R)} \int P_{\mathrm{g}, 1}(r) f(\theta, \varphi) \mathrm{d} l \\
& \simeq \frac{\rho_{\mathrm{g}, 0}^{\prime}(R)}{P_{\mathrm{g}, 0}^{\prime}(R)} y_{1}(R) m(\varphi) \\
& \simeq \frac{\rho_{\mathrm{g}, 0}^{\prime}(R)}{P_{\mathrm{g}, 0}^{\prime}(R)}\left(y(R, \varphi)-y_{0}(R)\right) .
\end{aligned}
$$

Here again we used the same notation and approximation as in Eq. (46). Note however that as soon as we assumed isothermality, the ratio $\rho_{\mathrm{g}, 0}^{\prime} / P_{\mathrm{g}, 0}^{\prime}$ is constant, therefore this last step is exact. Were we not assuming isothermality, the departure from isothermality is expected to be weak, thus this last approximation should be reasonable.

These last two alternative steps are crucial to our method since these approximations link the non spherically symmetric components of various quantities. They are reasonable, as will be discussed in Sect. 3.2.8 and will be numerically tested in Sect. 4 .

Of course, only well-known quantities appear in Eqs. (46) and (50): $y, y_{0}, \phi_{\mathrm{DM}}$ and $\phi_{0}$ are direct observational data whereas $P_{\mathrm{g}, 0}(r)$ and $\rho_{\mathrm{g}, 0}(r)$ are zeroth order quantities previously derived.

\subsection{7. ... and a projected gas temperature map to first order}

The projected temperature map can be obtained the same way as before. By using first the equation of state we can write :

$$
\begin{aligned}
& T_{\mathrm{g}, 0}(r)+\varepsilon T_{\mathrm{g}, 1}(r) f(\theta, \varphi)=\frac{1}{\beta} \frac{\left(P_{\mathrm{g}, 0}(r)+\varepsilon P_{\mathrm{g}, 1}(r) f(\theta, \varphi)\right)}{\left(\rho_{\mathrm{g}, 0}(r)+\varepsilon \rho_{\mathrm{g}, 1}(r) f(\theta, \varphi)\right)} \\
& \simeq \frac{1}{\beta}\left(\frac{P_{\mathrm{g}, 0}(r)}{\rho_{\mathrm{g}, 0}(r)}+\varepsilon P_{\mathrm{g}, 1}(r) \frac{\rho_{\mathrm{g}, 0}(r)-\rho_{\mathrm{g}, 1}(r)}{\rho_{\mathrm{g}, 0}^{2}(r)} f(\theta, \varphi)\right) \cdot(51
\end{aligned}
$$

Hence, since

$$
\begin{aligned}
\zeta(R, \varphi) & =\zeta_{0}(R, \varphi)+\varepsilon \zeta_{1}(R, \varphi) \\
& =\int T_{\mathrm{g}, 0}(r) \mathrm{d} l+\varepsilon \int T_{\mathrm{g}, 1}(r) f(\theta, \varphi) \mathrm{d} l
\end{aligned}
$$


we have

$\zeta_{1}(R, \varphi)=\int \frac{\rho_{\mathrm{g}, 0}(r)-\rho_{\mathrm{g}, 1}(r)}{\rho_{\mathrm{g}, 0}^{2}(r)} P_{\mathrm{g}, 1}(r) f(\theta, \varphi) \mathrm{d} l$.

Here we choose to approximate the last integral as previously discussed in order to make use of observational SZ data. Therefore we rewrite this last equation as:

$$
\begin{aligned}
& \zeta_{1}(R, \varphi) \simeq \frac{\rho_{\mathrm{g}, 0}(R)-\rho_{\mathrm{g}, 1}(R)}{\rho_{\mathrm{g}, 0}^{2}(R)} \int P_{\mathrm{g}, 1}(r) f(\theta, \varphi) \mathrm{d} l \\
& \simeq \frac{\rho_{\mathrm{g}, 0}(R)-\rho_{\mathrm{g}, 1}(R)}{\rho_{\mathrm{g}, 0}^{2}(R)} y_{1}(R) m(\varphi) \\
& \simeq \frac{\rho_{\mathrm{g}, 0}(R)-\rho_{\mathrm{g}, 1}(R)}{\rho_{\mathrm{g}, 0}^{2}(R)}\left(y(R, \varphi)-y_{0}(R)\right) .
\end{aligned}
$$

We obtain this way an expression to first order for the projected temperature in terms of either observed quantities or previously derived functions.

\subsubsection{Why the previous approximation is reasonable on intuitive grounds}

Our previous approximations can be justified on intuitive grounds even if we will take care in validating it numerically in Sect. 4 below. It relies on the fact that perturbations have by definition a finite extent, i.e. the first order correction to the perfectly circular (spherical) term is non zero only within a finite range. The typical size and amplitude of the perturbation can be easily scaled from the SZ and WL data set. This guarantees the validity of our assumptions on observational grounds. The key point is that the perturbation itself has a kind of axial symmetry, whose axis goes through the center of the cluster and the peak of the perturbation. This is reasonable if the perturbation originates in e.g. an incoming filament but not for a substructure. The latter would therefore have to be treated separately by superposition (see Sect. 5). This leads naturally to the statement that the typical angle we observe in the image plane is equal to the one we would observe if the line of sight were perpendicular to its actual direction, i.e. the perturbation as intrinsically the same angular extent in the directions along the line of sight and perpendicular to it. This is illustrated schematically in Fig. 2.

Given this description, we are now in a position to discuss the validity of our approximation. It consists of approximating the line of sight integral

$$
\int g(r) \Phi_{\mathrm{DM}, 1}(r) f(\theta, \varphi) \mathrm{d} l
$$

by

$$
g(R) \int \Phi_{\mathrm{DM}, 1}(r) f(\theta, \varphi) \mathrm{d} l
$$

where $g$ is any radial function. This approximation would be exact if $g(r)$ were constant in the relevant domain, i.e. if the line of sight had a constant $r$. As mentioned before, this is the case in Eq. (50) if we assume isothermality.
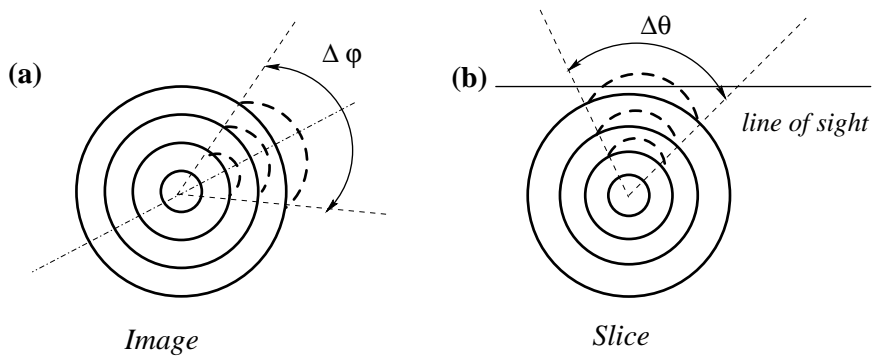

Fig. 2. We represent schematically in a) an image corresponding to our hypothesis. The full line corresponds to the perfectly circular $2-D$ term, e.g. $\phi_{\mathrm{DM}, 0}$, and the dashed line to the first perturbative correction to it, e.g. $\phi_{\mathrm{DM}, 1} m(\varphi), \Delta \varphi$ represents the observed angular extent. In $\mathbf{b}$ ) we represent a schematic slice in the $3-D$ potential responsible for this image. This slice has been performed along the dash-two-dotted plane indicated in figure a). Here again, the full line corresponds to the perfectly circular $3-D$ term, e.g. $\Phi_{\mathrm{DM}, 0}$, and the dashed line to the first perturbative correction to it, e.g. $\Phi_{\mathrm{DM}, 1} f(\theta, \varphi)$. The line of sight direction is indicated by the full thin line. Were the line of sight perpendicular to this slice plane, we would observe the angular extent $\Delta \theta$. Giving an axial symmetry to this perturbation leads us to assess that $\Delta \varphi \simeq \Delta \theta$.

However the functions $g(r)$ we might deal with may scale roughly as $r^{2}$, as e.g. $\rho_{\mathrm{g}, 0}^{\prime}(r) / P_{\mathrm{g}, 0}(r)$ in Eq. (46), thus it is far from being constant. The subsequent error induced can be estimated by the quantity $\Delta r g^{\prime}(r)$ where $\Delta r$ is the maximum $r$ discrepancy between the value assumed, $g(R)$, and the actual value as it is schematically illustrated in Fig. 3. In the worst case, $g^{\prime}(r)$ scales as $r$. Then, by using the obvious notations defined in this figure we get

$(\Delta r)_{\max }=R\left(1-1 / \sin \left(\theta-\frac{\Delta \theta}{2}\right)\right)$.

Naturally this quantity is minimal for $\theta \simeq 90^{\circ}$ and diverges for $\theta \simeq 0^{\circ}$ when $\Delta \theta=0^{\circ}$ : the error is minimal when the line of sight is nearly tangential $\left(\theta \simeq 90^{\circ}\right)$ and so is almost radial in this domain, and maximal when it is radial $\left(\theta=0^{\circ}\right)$. This in principle is a very bad behavior, but the fact is that the closer $\theta$ is to $0^{\text {deg }}$ the weaker the integrated perturbation is since it becomes always more degenerate along the line of sight, i.e. the integrated perturbations tend to a radial behavior and will therefore be absorbed in the $\Phi_{\mathrm{DM}, 0}(r)$ term. The extreme situation, i.e. when $\theta=0^{\mathrm{deg}}$, will trigger a mere radial image as long as the perturbation exhibits a kind of axial symmetry. This error is impossible to correct since we are dealing with a fully degenerate situation but it will not flaw the method at all since the integrated perturbation will be null. This approximation will be validated numerically below.

\subsection{How to obtain a X-ray prediction}

The previously derived map offers an advantage that we now aim to exploit, namely the ability of precise $\mathrm{X}$ 


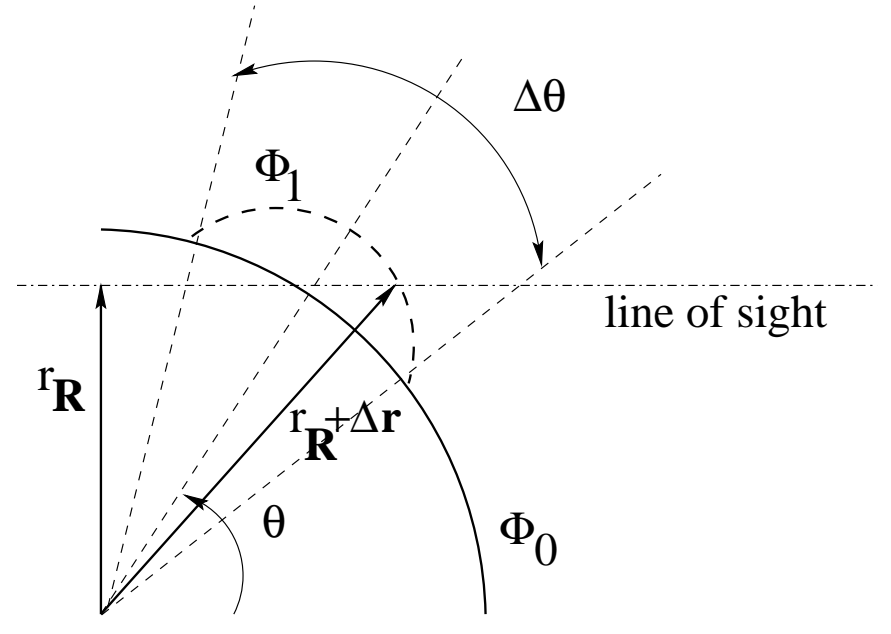

Fig. 3. We define in this figure the notation necessary to discuss our approximation. $r_{\mathrm{R}}$ is the parameter value given to the function $(f(r))_{\mathrm{R}}$ and $r_{\mathrm{R}}+\Delta r$ is an actual value along the line of sight.

prediction. Indeed, for a given X spectral emissivity model, the X-ray spectral surface brightness is

$S_{\mathrm{X}}(E)=\frac{1}{4 \pi(1+z)^{4}} \int n_{\mathrm{e}}^{2} \Lambda\left(E, T_{\mathrm{e}}\right) \mathrm{d} l$

where $\Lambda$ is the spectral emissivity, $z$ is the redshift of the cluster and $E$ is the energy on which the observed band is centered. Hence we can write, assuming a satisfying knowledge of $z$ and $\Lambda$ :

$$
\begin{aligned}
S_{\mathrm{X}}(E) & \propto \int n_{\mathrm{e}}^{2} T_{\mathrm{e}}^{1 / 2} \mathrm{~d} l \\
& \propto \int \rho_{\mathrm{g}}^{2} T_{\mathrm{g}}^{1 / 2} \mathrm{~d} l \\
& \propto \int \rho_{\mathrm{g}, 0}^{2} T_{\mathrm{g}, 0}^{1 / 2} \mathrm{~d} l+2 \varepsilon \int \rho_{\mathrm{g}, 0} T_{\mathrm{g}, 0}^{1 / 2} \rho_{\mathrm{g}, 1} f(\theta, \varphi) \mathrm{d} l \\
& +\frac{1}{2} \varepsilon \int \rho_{\mathrm{g}, 0}^{2} T_{\mathrm{g}, 0}^{-1 / 2} T_{\mathrm{g}, 1} f(\theta, \varphi) \mathrm{d} l
\end{aligned}
$$

where we omitted to write the $(r)$ s for clarity's sake. If we now make use of the same approximation as used and discussed before, we can express directly this quantity in terms of observations $y$ and $\phi$. We get indeed

$$
\begin{aligned}
S_{\mathrm{X}}(E) \propto & \int \rho_{\mathrm{g}, 0}^{2} T_{\mathrm{g}, 0}^{1 / 2} \mathrm{~d} l \\
& +2 \varepsilon \rho_{\mathrm{g}, 0}(R) T_{\mathrm{g}, 0}^{1 / 2}(R) \int \rho_{\mathrm{g}, 1} f(\theta, \varphi) \mathrm{d} l \\
& +\frac{1}{2} \varepsilon \rho_{\mathrm{g}, 0}^{2}(R) T_{\mathrm{g}, 0}^{-1 / 2}(R) \int T_{\mathrm{g}, 1} f(\theta, \varphi) \\
\propto & \int \rho_{\mathrm{g}, 0}^{2} T_{\mathrm{g}, 0}^{1 / 2} \mathrm{~d} l+2 \varepsilon \rho_{\mathrm{g}, 0}(R) T_{\mathrm{g}, 0}^{1 / 2}(R) D_{\mathrm{g}, 1}(R, \varphi) \\
& +\frac{1}{2} \varepsilon \rho_{\mathrm{g}, 0}^{2}(R) T_{\mathrm{g}, 0}^{-1 / 2}(R) \zeta_{\mathrm{g}, 1}(R, \varphi) .
\end{aligned}
$$

Both the zeroth order terms $T_{\mathrm{g}, 0}$ and $\rho_{\mathrm{g}, 0}$, and the first order corrections $D_{\mathrm{g}, 1}$ and $\zeta_{\mathrm{g}, 1}$ have been derived in the previous sections. We are thus able to generate selfconsistently a X luminosity map from our previously derived maps. This is a very nice feature of this method. We will further discuss the approximation and its potential bias in the next section.

This derivation opens the possibility of comparing on the one hand SZ and WL observations with, on the other hand, precise X-ray measurements as taken e.g. by XMM or CHANDRA. Note that in the instrumental bands of most X-ray satellites the $T_{\mathrm{g}}$ dependence is very weak and can be neglected. This can be easily taken into account by eliminating the $T_{\mathrm{g}}$ dependence in the previous formula. Even if the interest of such a new comparison is obvious, we will discuss it more carefully in the two following sections. In principle, one could also easily make some predictions concerning the density weighted X-ray temperature defined by the ratio $\int n_{\mathrm{g}}^{2} T_{\mathrm{g}} \mathrm{d} l / \int n_{\mathrm{g}}^{2} \mathrm{~d} l$ but the fact is that since the gas pressure and so the SZ effect tends to have a very weak gradient, we are not able in principle to reproduce all the interesting features of this quantity, namely the presence of shocks.

\section{Application on simulations}

In order to demonstrate the ability of the method in a simplified context we used some outputs of the recently developed $N$-body + hydrodynamics code RAMSES simulating the evolution of a $\Lambda$-CDM universe. The RAMSES code is based on Adaptative Mesh Refinement (AMR) technique in order to increase the spatial resolution locally using a tree of recursively nested cells of smaller and smaller size. It reaches a formal resolution of $12 h^{-1} \mathrm{kpc}$ in the core of galaxy clusters (see Réfrégier \& Teyssier 2000 and Teyssier 2001 , in preparation, for details). We use here the structure of two galaxy clusters extracted from the simulation to generate our input observables, i.e. X-ray emissivity, SZ decrement and projected mass density (or projected gravitational potential).

The relevant observables, i.e. projected mass density, SZ decrement and, for comparison purpose only, the Xray emission measure, of the 2 clusters are depicted using a logarithmic scaling in Figs. 4 and 5 (upper panels). These clusters have been extracted from the simulation at $z=0.0$ and thus tend to be more relaxed than high $z$ ones: they are ordinary clusters of virial mass (defined by $\delta_{334}$ in our particular cosmology) $4.50 \times 10^{14} h^{-1} M_{\odot}$ and $4.15 \times 10^{14} h^{-1} M_{\odot}$. Both exhibit a rather regular shape, i.e. they have not recently undergone a major merging process. The depicted boxes are respectively $3.5 h^{-1} \mathrm{Mpc}$ and $4.0 h^{-1} \mathrm{Mpc}$ wide. We smooth the outputs using a Gaussian of width $120 h^{-1} \mathrm{kpc}$ thus degrading the resolution. We did not introduce any instrumental noise. For both clusters, the gas temperature decreases by a factor of less than 2 from the center to the virial radius. Beyond, the temperature profile is steeper. Nevertheless, for the sake of simplicity we will assume that $T_{\mathrm{g}}$ is constant, making the discussion of $T_{\mathrm{g}, 0}$ and $T_{\mathrm{g}, 1}$ useless at this point. 

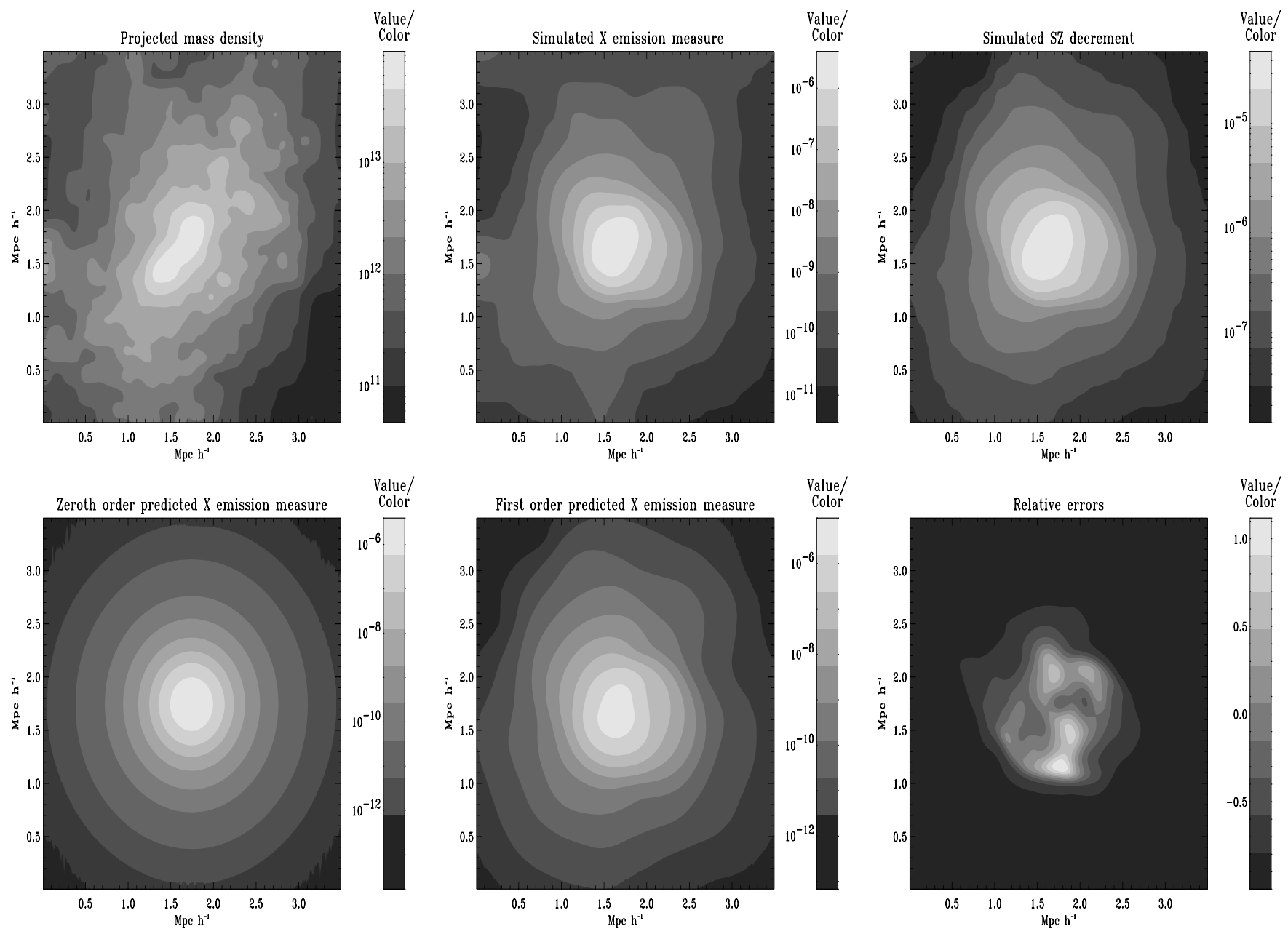

Fig. 4. The upper panel shows the results of simulations, from left to right, all using a logarithmic scaling, the projected mass density $\left(M_{\odot} \mathrm{Mpc}^{-2}\right)$, the X-ray emission measure $\left(\mathrm{cm}^{-6} \mathrm{Mpc}\right)$ and the SZ $y$ parameter. This cluster is a good candidate for our approach since it has a circular core with surrounding perturbations so would be inappropriate for a ellipsoidal fit. The lower panel shows, from left to right a zeroth order predicted X emission measure, the first order prediction (the zeroth order term plus the first order correction), both using a logarithmic scaling as well as the the relative error map, i.e. (predicted simulated)/simulated $\mathrm{X}$ emission measure using a linear scaling. The 10 error contours are linearly separated between -1.0 and 1. Each box is $3.5 \mathrm{~h}^{-1} \mathrm{Mpc}$ wide. The correlation coefficient between the predicted and the simulated X-ray emission measure is 0.978 . The total fluxes differ only by $0.91 \%$, thus even if the relative error map increases at high $R$ the total error remains small due to the great dynamical range involved.

We apply the method previously described using perturbed spherical symmetry. We define the pixel with the highest luminosity to be the center. The zeroth order circular description of the gas density is computed averaging data over concentric annuli and we then add to it some first order corrections. Note that since we assume isothermality, SZ data give us straightforwardly a projected gas density modulo a temperature $T_{\mathrm{g}, 0}$ coefficient, thus we use the formulation of Eq. (50), which is exact in this context. This constant temperature is fixed using the hydrostatic equilibrium and the WL data.

In Figs. 4 and 5 (lower panels) we show the predicted $\mathrm{X}$-ray emission measure to zeroth and first order as well as a map of relative errors. Note that to first order the shape of the emission measure is very well reproduced. The crosscorrelation coefficients between the predicted and simulated X-ray emission measures are 0.978 and 0.986 . Of course this is partly due to the assumed good quality of the SZ data but nonetheless, it demonstrates the validity of our perturbative approach as well as of our approximation. The approximation performed in Eq. (65), i.e. the multiplication by the function $\rho_{\mathrm{g}, 0}(R)$ naturally tends to cut out the perturbations at high $R$. This is the reason why the further perturbations are slightly less well reproduced and the relative errors tend to increase with $R$. Nevertheless, since the emission falls rapidly with $R$, as is visible in the lower figures (note the logarithmic scaling), the total flux is well conserved, respectively to $0.9 \%$ and $9 \%$. This last number might illustrate that the large extent of the perturbations in the second case may limit our method. An ellipsoidal fit could help decrease this value. Note that the clump visible mainly in X-ray emission measure in 5 is not reproduced. This is natural because it does not appear through the SZ effect since the pressure remains uniform 



Fig. 5. As in the previous figure for a different cluster. The structure of the X-ray emission measure is very well reproduced for the inner part. The correlation coefficient between the predicted and simulated map is 0.986 . As is visible on the relative error map, whose 10 levels are linearly separated between -1.0 and 1.0, the outer part is naturally smeared by our approximation. The visible clump on the top-right should be treated separately. Each box is $4.0 \mathrm{~h}^{-1} \mathrm{Mpc}$ wide. The total flux differs by $9 \%$.

throughout the clumps. If resolved by WL, this substructure should be treated separately, e.g. by considering the addition of a second very small structure. Note that the first cluster showed exhibits a spherical core elongated in the outer region, thus it is not actually as ellipsoidal as it looks, which may explain why our perturbed spherical symmetry works well.

\section{Discussion}

\subsection{Hypotheses ... and non hypotheses}

The general and robust hypotheses have been introduced and discussed in Sect. 2.1. Our key hypothesis consists of assuming the validity of a perturbative approach and in choosing the nature of this perturbation, i.e. with a radial/angular separation. It was initially motivated by theoretical predictions, observations and simulations which show that relaxed clusters are regular and globally spheroidal objects. In fact, comparison of our models with simulated clusters confirms that our assumptions turn out be reasonable. However, our method cannot deal properly with sharp features e.g. shocks waves due to infalling filaments. Assuming the validity of the angular and radial separation implies the equality of this angular part for all relevant physical quantities $\left(P_{\mathrm{g}}, T_{\mathrm{g}}, \phi_{\mathrm{DM}} \ldots\right)$, using to a first order in $\varepsilon$ the hydrostatic equilibrium and the equation of state. If this is not satisfied in practice, then we could question the validity of this separation or of the underlying physics introduced in the cluster model. Our experience with simulations shows that for reasonably relaxed clusters which do not experience a major merging process, the angular part of the perturbation is constant amongst observables. In that case, the separation (and thus the equality of the angular perturbation) seems to be a good hypothesis in general, whereas its failure is a sign of non-relaxation, i.e. non-validity of our general physical hypothesis.

The validity of our approximations also depends on physical quantities which are assumed to be constant along the integral. In the case of the gas density obtained 
from the SZ map, it is an exact statement, provided we assume isothermality. Since clusters in general are not too far from isothermality, this hypothesis is reasonable.

The strength of the perturbation method we propose is the fact that we do not need to assume isothermality and sphericity (or ellipsoidality). This might be of importance for further applications. An interesting example is the measurement of the Hubble constant from joint SZ and X-ray measurements. As it has been stressed by Inagaki et al. (1995); Roettiger et al. (1997); Puy et al. (2000), both the asphericity and the non-isothermality of the relevant clusters can yield important biases in the estimate of $H_{0}$ (up to $20 \%$ ). Our approach may be relevant to address this issue.

Although the comparaison with simulated clusters is conclusive, our perturbative method has two shortcomings:

- We implicitly assume an equivalent degree of asymmetry for the physical quantities involved, i.e. mainly the gas pressure and DM potential. If perturbations of spherical models is produced by incoming filaments, as discussed above, this assumption is reasonable. However, this particular point might require more detailed study.

- We obtain good results by assuming an isothermal distribution. In fact, although the difference is small, it turns out that the underlying distribution is closer to a polytropic one (compatible with the results of Markevitch 1998). This means that the overall baryonic content is well approximated if we assume a (wrong) isothermal profile but that we would fail to reproduce precisely the underlying distributions, even if the total mass of each components is very well estimated.

Note finally that in the previous sections, we chose to predict the X-ray brightness map from SZ and WL data instead of predicting SZ maps from WL and X-ray. Since X-ray maps have higher resolution observations than SZ or WL, this way to proceed may be questionnable (this situation will remain unchanged even with future SZ experiments). In fact, SZ is the most robust observable. It only depends on the pressure, which is a very smoothly varying quantity and its emission model is fairly simple, in contrast to the X-ray one. Moreover, it probes better the outer part of the cluster, i.e. it is less concentrated than the X-ray emission.

\subsection{The equivalent spheroidal symmetry case}

So far, we have discussed the perturbed spherical symmetry case. If we turn to spheroidal symmetry, the problem is similar provided we know the inclination angle $i$ between the polar axis of the system and the line of sight. In the appendix we review the basic results of spheriodal deprojections. It is directly inspired from (Fabricant et al. 1984): once the projection is well parameterized we get the projected quantity, like the pressure:

$$
\begin{aligned}
y(\eta) & =2 \frac{B_{\mathrm{e}}}{R} \int_{\eta}^{\infty} \frac{P_{\mathrm{g}, 0}(t) t \mathrm{~d} t}{\left(t^{2}-\eta^{2}\right)^{\frac{1}{2}}} \\
P_{\mathrm{g}, 0}(t) & =-\frac{1}{2 \pi} \frac{R}{B_{\mathrm{e}}} \int_{t}^{\infty} P_{\mathrm{g}, 0}^{\prime}(\eta) \frac{\mathrm{d} \eta}{\left(\eta^{2}-t^{2}\right)^{1 / 2}},
\end{aligned}
$$

following the notations of the appendix. Since we are dealing with the same Abel integral we can proceed in two steps as we did before.

Even if the inclination angle is a priori not accessible directly through single observations, it is possible to evaluate it using the deprojection of an axially symmetric distribution of either X-ray/SZ maps or SZ/surface density maps (Zaroubi et al. 1998; Zaroubi et al. 2000). Our method overcomes this problem since it only focusses on 2-D quantities and avoids as much as possible to deal with the full 3-D structure. However, in principle the method can also reconstruct $3-D$ quantities. This will be discussed in a forthcoming work. Note also that axially symmetric configuration elongated along the line of sight may appear spherical. This is a difficult bias to alleviate without any prior knowledge of the profile. In our case, our method will be biased in the sense that the deprojected profile will be wrong. Nevertheless, we might hope to reproduce properly global quantities, like abundance of DM or gas and so to alleviate some well known systematics (see previous section), e.g. in measuring the baryon fraction.

\subsection{Application to true data}

When applying the method to true data, the instrumental noise issue is an important matter of concern. The distinct advantage of a parametric approach, e.g. using a $\beta$-model, is that it fits the relevant parameters, e.g. $r_{\mathrm{c}}$ and $\beta$, on the projected quantities (the image) itself, which is rather insensitive to noise. However, it might be difficult to determine the profile and its derivate by a direct deprojection. In our perturbative approach, as it first relies on a zeroth order quantity found by averaging over some annulus, a noise reduction step (at least far from the center) prior to working on a projected perturbation should be quite robust as well. Nevertheless, this problem is not critical and can be addressed easily by using a more sophisticated technique than the mere use of Abel's integral. In particular we could decompose the profile into a function basis whose projection is known analytically (see for example Durret et al. 1999 in another context).

\section{Conclusion}

Trying to answer the question of how to coanalyse a set of WL and SZ data, we have presented and demonstrated the efficiency of an original method allowing us to perform in a self-consistent manner the joint analysis of these data. Using it on noise-free simulations, we demonstrated how well it can be used to make predictions of X-ray surface brightness. We discussed also in the previous sections 
some shortcomings that do not seem critical but definitely require further work.

Compared to other, we choose to hide the deprojection by using appropriate approximations. Thus, we do not resolved fully the $3-D$ structure of clusters, although this work is a first step towards a full deprojection (Doré et al. 2001 , in preparation).

Nevertheless, since it is based on a self-consistent use of general physical hypotheses, the X-ray emission prediction of our method when compared to the observed one constitutes an interesting test of these hypotheses, namely the stationarity, the hydrostatic equilibrium and the validity of the equation of state.

This method is furthermore of interest since it should allow a better treatment of systematics (asphericity, non isothermality, ...) plaguing any measure of the baryon fraction $f_{\mathrm{b}}$ or the Hubble constant $H_{0}$ using X-ray and SZ the effect (Inagaki et al. 1995). Thus we plan to apply it soon on true data.

Acknowledgements. We are grateful to our referee, M. Bartelmann, and to S. Dos Santos, G. Mamon and S. Zaroubi for valuable discussions. We thank J. Calstrom et al. for allowing the use of some of their SZ images.

\section{Appendix: Deprojection in spheroidal symmetry}

In this appendix we recall some useful results concerning spheroid projection derived by Fabricant, Gorenstein and Rybicki (Fabricant et al. 1984). In the context of spheroidal systems, cartesian coordinates system are the most convenient for projection. Thus, if the observer's coordinate system $(x, y, z)$ is chosen such that the line of sight is along the $z$ axis and such that the polar axis of the spheroidal system $z^{\prime}$ lies in the $x-z$ plane at an inclination angle $i$ to the $z$-axis, then, in the cartesian coordinate system $\left(x^{\prime}, y^{\prime}, z^{\prime}\right)$ the general physical quantities relevant to our problem depends only on the parameter $t$ defined by

$$
\begin{aligned}
t^{2} & =\frac{x^{\prime 2}+y^{\prime 2}}{B_{\mathrm{e}}^{2}}+\frac{z^{\prime 2}}{A_{\mathrm{e}}^{2}} \\
& =\frac{(x \cos i+y z \sin i)^{2}+y^{2}}{B_{\mathrm{e}}^{2}}+\frac{(z \cos i-x \sin i)^{2}}{A_{\mathrm{e}}^{2}} .
\end{aligned}
$$

If we project a physical quantity $G(t)$ on the observer sky plane $x-y$ then,

$$
\begin{aligned}
I(x, y) & =I(\eta) \\
& =\int_{-\infty}^{+\infty} G(t) \mathrm{d} l \\
& =2 \frac{B_{\mathrm{e}}}{R} \int_{\eta}^{\infty} \frac{G(t) t \mathrm{~d} t}{\left(t^{2}-\eta^{2}\right)^{\frac{1}{2}}}
\end{aligned}
$$

where

$$
\eta^{2} \equiv \frac{x^{2}}{\left(R A_{\mathrm{e}}\right)^{2}}+\frac{y^{2}}{\left(B_{\mathrm{e}}\right)^{2}}
$$

and $R \equiv \sqrt{\frac{B_{\mathrm{e}}^{2}}{A_{\mathrm{e}}^{2}} \cos ^{2} i+\sin ^{2} i}$.

Of course this result shows that if we were to observe a spheroidal system we would map ellipses with an axial ratio equal to $\frac{B}{A}=\frac{1}{R} \frac{B_{\mathrm{e}}}{A_{\mathrm{e}}}$. But the main result of this appendix is that we obtain at the end an Abel integral similar to the one obtained in the case of spherical system, where the radius as been replaced by the parameter $t$. This simple fact justifies the very analogous treatment developed in this paper for spherical and spheroidal systems.

\section{References}

Bartelmann, M., \& Schneider, P. 2001, Phys. Rep., 340, 291

Binney, J., \& Tremaine, S. 1987 (Princeton University Press)

Birkinshaw, M. 1999, Phys. Rep., 310, 97

Cavaliere, A., \& Fusco-Femaino, R. 1976, A\&A, 49, 137

Castander, F. J., et al. 2000, in Constructing the Universe with clusters of galaxies, ed. F. Durret, \& D. Gerbal

Chièze, J., Alimi, J. \& Teyssier, R. 1998, ApJ, 495, 630

Désert, F.-X., et al. 1998, New Astron., 3, 655

Doré, .O., et al. 2000, in Constructing the Universe with clusters of galaxies, ed. F. Durret, \& D. Gerbal

Durret, F., Gerbal, D., Lobo, C., \& Pichon, C. 1999, A\&A, 343,760

Fabian, A. C., et al. 1981, ApJ, 248, 47

Fabricant, D. Rybicki, G., \& Gorenstein, P. 1984, ApJ, 286, 186

Holder, G., \& Carlstrom, J. 1999, in Microwave Foregrounds, ed. A. de Oliveira-Costa, \& M. Tegmark, ASP Conf. Ser. 181

Holder, G., et al. 2000, in Constructing the Universe with clusters of galaxies, ed. F. Durret, \& D. Gerbal

Grego, L., et al. 2000, ApJ, 539, 39

Inagaki, Y., Suginohara, T., \& Suto, Y. 1995, PASJ, 47, 411

Markevitch, M. 1998, ApJ, 504, 27

Mellier, Y. 2000, ARA\&A, 37, 127

Puy, D., et al. [astro-ph/0009114]

Rephaeli, Y. 1995, ARA\&A, 33, 541

Reblinsky, K., \& Bartelmann, M. [astro-ph/9909155]

Reblinsky, K. 2000, Ph.D. Thesis at Ludwig Maximilians Universität München

Reese, E. D., et al. 2000, ApJ, 533, 38

Refregier, A., \& Teyssier, R., submitted to Phys. Rev. D [astro-ph/0012086]

Roettiger, K., Stone, J., \& Mushotzky, R. F. 1997, ApJ, 482, 588

Sarazin, C. L. 1988, X-ray emission from clusters of galaxies (Cambridge University Press)

Sunyaev, R., \& Zel'dovich, I. 1972, Comments Astrophys. Space Phys., 4, 173

Sunyaev, R., \& Zel'dovich, I. 1980, ARA\&A, 18, 537

Teyssier, R., Chièze, R., \& Alimi, J. 1997, ApJ, 480, 36

Yoshikawa, K., \& Suto, Y. 1999, ApJ, 513, 549

Zaroubi, S., et al. 1998, ApJ, 500, L87

Zaroubi, S., et al. [astro-ph/0010508]

Zel'dovich, I., \& Sunyaev, R. 1969, Astrophys. Space Sci., 4, 301 